\title{
Predictors of mortality following extracorporeal membrane oxygenation support in an unselected, critically ill patient population
}

\author{
István Ferenc Édes ${ }^{1}$, Balázs Tamás Németh ${ }^{1}$, István Hartyánszky ${ }^{1}$, Bálint Szilveszter ${ }^{1}$, Péter Kulyassa ${ }^{1}$, \\ Levente Fazekas ${ }^{1}$, Miklós Pólos ${ }^{1}$, Endre Németh ${ }^{2}$, Dávid Becker ${ }^{1}$, Béla Merkely ${ }^{1}$ \\ ${ }^{1}$ Heart and Vascular Center, Semmelweis University, Budapest, Hungary \\ ${ }^{2}$ Department of Anesthesiology and Intensive Therapy, Semmelweis University, Budapest, Hungary
}

Adv Interv Cardiol 2021; 17, 3 (65): 290-297

DOI: https://doi.org/10.5114/aic.2021.109149

\begin{abstract}
Introduction: Mechanical circulatory support (MCS) has been established as a means of augmenting circulation in patients with critically decreased systolic function due to a variety of underlying clinical reasons. Different methods of MCS may be used, with the venous-arterial extracorporeal membrane oxygenation system (VA-ECMO) being one of the most utilized devices in everyday care.

Aim: To determine independent predictors influencing mortality outcomes following VA-ECMO therapy in a large, unselected, adult, critically ill patient population in cardiogenic shock (CS).

Material and methods: Data on 235 consecutive, real-world VA-ECMO treatments were assessed. Analysis was conducted for all subjects requiring MCS with the VA-ECMO as the first instalment, regardless of underlying cause or eventual upgrade. All potential clinical factors influencing mortality were examined and evaluated.

Results: Overall mortality was $\sim 66 \%$ at median 28 days follow-up and significantly depended upon $\mathrm{pH}<7.3(\mathrm{HR}=3.56$; $p<0.001)$, and age $\geq 65$ years ( $H R=1.96 ; p=0.001)$. Acute coronary syndrome (ACS) as an indication for VA-ECMO displayed a nearly significant value $(H R=1.44 ; p=0.07)$. Heart transplant ( $\mathrm{hTX}$ ) primary graft failure as an indication for the VA-ECMO displayed a clearly favorable outcome ( $\mathrm{HR}=0.51, p=0.025)$; all data based on multivariate Cox regression analysis.

Conclusions: Mortality in patients requiring VA-ECMO remains high. We conclude that only decreased pH values and advanced age clearly influence mortality in this MCS scenario. ACS also bodes unfavorably, whereas hTX as an indication clearly shows better survival.
\end{abstract}

Key words: survival, mechanical circulatory support, pVA-ECMO.

Su m m a ry

To further our understanding on veno-arterial extracorporeal membrane oxygenator (ECMO) support and outcomes we investigated independent predictors of mortality in refractory cardiogenic shock (CS) patients requiring ECMO support, regardless of the cause of CS. We managed to gather 7 years of data and to the best of our knowledge the largest patient cohort (235 subjects) from a national level ECMO center. As such, we evaluated ECMO outcomes from a completely new perspective, thus providing exciting new results, relevant to everyday medical practice.

\section{Introduction}

Mechanical circulatory support (MCS), also known as extracorporeal life support, is a means of augmenting or even fully providing circulation for patients with severely diminished cardiac systolic function. Besides establishing effective cardiac output, some MCS strategies allow for oxygenation of the patients' blood via an external method, using a capillary-based oxygenator matrix. MCS indications, implantation strategies and methods vary greatly in utility, ease of use and treatment length. One of the most common short-term forms of MCS for systemic circulation enhancement is the venous-arterial (VA) extracorporeal membrane oxygenation (ECMO) system, which

\section{Corresponding author:}

István Ferenc Édes Dr. PhD, Heart and Vascular Center, Semmelweis University, Budapest, Hungary, phone: +36-20-663-2488,

e-mail: edes789@gmail.com

Received: 21.05.2021, accepted: 18.06.2021. 
has proven to be efficient to establish and operate [1, 2]. VA-ECMO systems have evolved to a contemporary method of fast and effective circulatory enhancement, which may be implemented rapidly should the requirement arise [3].

Over the years, VA-ECMO utilization has broadened in indications for short-term (7-14 days) use and is currently employed in a variety of conditions leading to depressed circulation and ventilation refractory to lesser forms of circulatory support. Systemic circulation may be augmented from a central or peripheral approach. The central ECMO system is mostly implemented in conjunction with open-heart surgery as it requires a sternotomy and direct cannulation of the ascending aorta and right atrium. In emergency or life-threatening conditions outside an operating theatre the peripheral approach is used, initiation of which may be performed in an out-of-cath lab, or even out-of-hospital, setting via cannulation of major veins and arteries outside the thoracic cavity. As various parameters, including treatment length or potential risks and benefits of peripheral and central ECMO, differ, upgrade to central cannulation may be performed if required by the clinical course $[4,5]$. Also, further upgrade of either ECMO system is possible to mid- or long term ventricular assist devices (VAD), should the need arise $[6,7]$.

A sizeable cohort of ECMO patients has recently been analyzed utilizing mainly external data from an international registry, leading to the development of the SAVE score [8], which was validated in a matched cohort, thus establishing a novel means of predicting ECMO outcomes [8].

\section{Aim}

Since to the best of our knowledge, data from extended databases of national level centers utilizing the technology in diverse real-world scenarios are lacking, we set forth to clarify independent risk factors influencing mortality associated with VA-ECMO treatment, irrespective of indication and underlying pathology based on our patient population.

\section{Material and methods}

VA-ECMO patients, endpoints and follow-up

Data on all MCS procedures utilizing the VA-ECMO as a bridge-to-recovery or bridge-to-bridge strategy at our institution have been consecutively collected since the instigation of our first case in 2012. Our database analyzed in the current study encompasses 235 VA-ECMO cases ending in October, 2020 (Figure 1). Patients undergoing VA-ECMO treatment were in cardiogenic shock (CS), defined as decreased cardiac output and/or myocardial contractility resulting in generalized hypoperfusion, refractory to non-MCS treatment [9], and were on Interagency Registry for Mechanically Assisted Circulatory Support (INTERMACS) level 1 [10]. Since initiation of our MCS program, the number of VA-ECMO treatments has been steadily rising (Figure 1). Demographics, co-morbidities, clinical features, underlying medical causes of VA-ECMO initiation and implantation details, any system upgrades, laboratory and imaging findings, complications and mortality events have been recorded and pooled (Table I).

Due to the nature of this critically, often terminally ill patient population, we assessed independent predictors influencing all-cause mortality following ECMO treatment as our only endpoint in the register. The Regional and Institutional Committee of Science and Research Ethics of Semmelweis University evaluated our registry and gave its approval (reference number: 82/2019). Follow-up of patients surviving initial care was clinically driven and the results were verified using the Hungarian National Health Insurance database.

\section{Indications for VA-ECMO implantation, other devices}

Underlying medical conditions of CS necessitating VA-ECMO implantation varied over a wide spectrum (Table I), the three most prevalent causes being acute coronary syndrome (ACS) associated CS, primary graft failure following heart transplantation (hTX) and post-cardiotomy CS. Severe and rapid acute decompensation of chronic heart failure leading to circulatory collapse and fulminant myocarditis were also of note. Other, infrequent causes comprising altogether $6.4 \%$ of VA-ECMO implantations were as follows: propafenone intoxication, complicated percutaneous valve interventions, electrophysiological ablations, pacemaker electrode and generator explants, interventional radiological procedures and circulatory bridging necessity due to sudden long-term left-ventricular assist device (LVAD) failure until emergency redo surgery (Table I). Other forms of circulatory support alongside the VA-ECMO providing a pulsatile

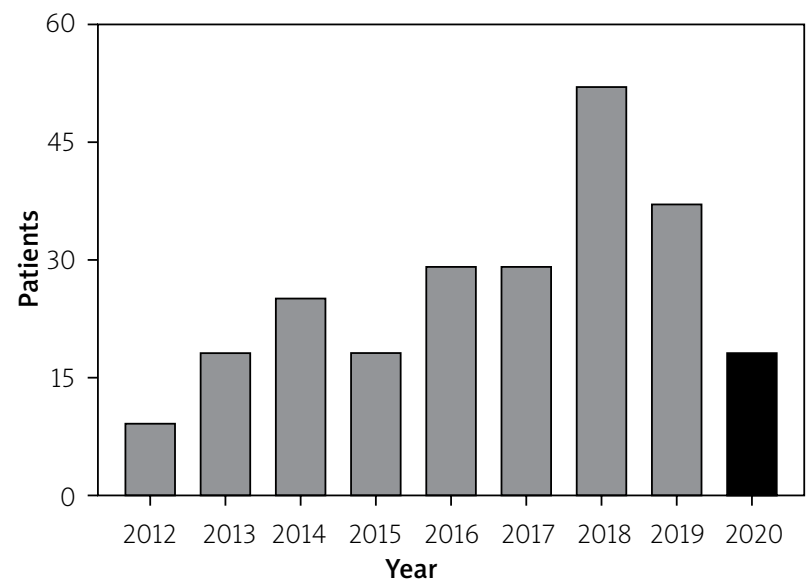

Figure 1. Number of venous-arterial extracorporeal membrane oxygenation (VA-ECMO) implantations since the initial case in 2012. Data collection of exactly 235 patients ended with the last day of October 2020 
Table I. VA-ECMO implantation indications, techniques, concomitant procedures, specifics. Study population $(n=235)$

\begin{tabular}{lc}
\hline Patient data & Value \\
\hline VA-ECMO implantation due to: $n$ (\%): & \\
\hline ACS in manifest cardiogenic shock & $96(40.8)$ \\
\hline Primary graft failure after hTX & $50(21.3)$ \\
\hline Post-cardiotomy shock & $46(23.8)$ \\
\hline Acute decompensation of chronic DCM & $22(8.8)$ \\
\hline Fulminant myocarditis & $6(2.5)$ \\
\hline Other & $15(6.4)$ \\
\hline VA-ECMO techniques, $n$ (\%): & $133(56.5)$ \\
\hline Central cannulation & $102(43.5)$ \\
\hline Peripheral cannulation & $5.0 \pm 4.8$ \\
\hline VA-ECMO treatment duration [days] & $24(23.5)$ \\
\hline Backflow cannula inserted, pECMO only, $n$ (\%) & $157(66.8)$ \\
\hline LV decompression performed, $n$ (\%) & $7(6.8)$ \\
\hline Lower limb ischemia, pECMO only, $n(\%)$ & $33(14.0)$ \\
\hline Major bleeding events, $n$ (\%) & $102(43.4)$ \\
\hline Concomitant procedures undertaken, $n$ (\%): & $12(5.1)$ \\
\hline Percutaneous coronary intervention & $21(11.2)$ \\
\hline New hTX after VA-ECMO care & $32(13.6)$ \\
\hline Upgrade from peripheral to central cannulation & $216(91.9)$ \\
\hline Upgrade to mid/long-term LVAD & $10(4.2)$ \\
\hline Type of VA-ECMO system used, $n$ (\%): & $5(1.7)$ \\
\hline MEDOS & Centrimag \\
\hline BioMedicus & CardioHelp
\end{tabular}

ACS - acute coronary syndrome, DCM - dilative cardiomyopathy, hTX - heart transplantation, $L V A D$ - left ventricular assist device, $p E C M O$ - peripheral extracorporeal membrane oxygenation, VA-ECMO - venous-arterial extracorporeal membrane oxygenation. * See the text.

arterial pressure-wave, such as the intra-aortic balloon pump or direct short-term percutaneous heart pump devices such as the Impella or iVAC systems, were not used in our cohort. Out-of-hospital cardiac arrest (OHCA) patients in whom CPR was undertaken in conjunction with VA-ECMO support in the field (eCPR) were not included in the database. All MCS cannulations were performed in a medical hospital setting after initial transportation.

VA-ECMO cannulation, upgrades, left ventricular decompression, concomitant procedures, treatment length and systems

Cannulation approaches of systems depended mainly on initial procedures prior to MCS initiation. Central cannula implantation was mostly in conjunction with open heart surgery, while CS ACS patients initially received peripheral systems. Upgrades of running systems, either from peripheral to central VA-ECMO (21 cases $-11.2 \%)$, or any type of VA-ECMO to mid or long-term LVAD systems (32 cases $-13.6 \%$ ) were clinically driven when re-cannulation or longer-term circulatory support was required. Overall, including the cases that were upgraded from peripheral systems, the central approach was utilized slightly more often at $56.5 \%$. Left ventricular (LV) decompression was performed in $66.8 \%$ of all cases, with surgical methods (atrial shunt implantation) during CECMO and interventional methods (balloon atrial septostomy, or direct decompression via a left ventricular pigtail catheter) in pECMO cases. Percutaneous revascularization was clinically indicated and carried out in 102 (43.4\%) cases. Of all subjects requiring ECMO support, eventually 12 (5.1\%) subjects received a successful high-urgent heart transplantation. Length of circulatory augmentation (mean \pm standard deviation; 5.0 \pm 3.4 days) was mainly influenced by (I) clinical response of patients to MCS, (II) age and/or (III) clinical situations requiring cannula upgrades or device modification such as decline of the system oxygenator or arising clinical complications comprising mostly lower limb ischemia during peripheral VA-ECMO use and various degrees of bleeding from the puncture sites. Lower limb ischemia is a common complication with the arterial cannula of the peripheral VA-ECMO system. Backflow cannula insertion, involving an ipsilateral anterograde arterial puncture of the common femoral artery, is the method of choice to alleviate this issue and provide circulation to the lower limb [11-13]. Potential lower limb ischemia was evaluated angiographically and/or via the Doppler ultrasound method in our registry. In cases presenting with impeded anterograde flow, 7 French backflow sheaths were inserted using ultrasound guidance. These were used in $23.5 \%$ of peripheral VA-ECMO cases (Table I). Severe lower-limb ischemia was present in less than $6.8 \%$ of peripheral VA-ECMO cases. Major bleeding occurred in $14 \%$ of patients.

\section{Statistical analysis}

Continuous variables were expressed as mean \pm standard deviation or median with interquartile ranges, whereas categorical variables were expressed as percentages. Survival curves were plotted utilizing the Kaplan-Meier method. We used uni- and multivariate Cox regression analysis to evaluate the effects of the individual studied parameters on mortality. Determination of these risk factors was carried out by selecting variables with probabilities of $p<0.1$ from the univariate Cox regression analysis. These were further examined using the backwards stepwise multivariate regression model to assess independence from one another. The internationally accepted probability $(p<0.05)$ was chosen as the level of significance. Hazard ratios (HR) were also calculated, using a confidence interval $(\mathrm{Cl})$ of $95 \%$. In the receiver operating characteristics (ROC) curve analysis a lactate value of 3.3 and a $\mathrm{pH}$ value of 7.3 were used as an optimal cut-off point for discriminating the endpoint event (all-cause mortality). Data were stored and organized in 
Microsoft Excel 2016 and analyzed using the SPSS statistical software (version 23).

\section{Results}

\section{Demographics and clinical data}

Table II displays all relevant clinical and demographic data for this critically ill patient population. It is notable that subjects were relatively young ( $52.7 \pm 15.7$ years). Relevant cardiovascular co-morbidities, such as age $\geq 65$ years, diabetes and body mass index values exceeding $30 \mathrm{~kg} / \mathrm{m}^{2}$, all occurred at a lower rate than what is generally observed in classical cardiovascular clinical scenarios, with these factors prevalent in approximately $20-25 \%$ of the total population. The patients were predominantly male $(74 \%, n=174)$. Prior coronary revascularization was present in $21.2 \%(n=50)$ of cases, while patients with prior ACS represented $20.4 \%(n=48)$.

Both renal and left ventricular function was impaired in the current population. Glomerular filtration rate (GFR) was calculated using the Cockcroft-Gault formula, averaging $50.0 \pm 24.7 \mathrm{ml} / \mathrm{min} / 1.73 \mathrm{~m}^{2}$. Ejection fraction was measured in each subject as soon as possible after initiation of VA-ECMO, utilizing the bi-plane Simpson technique, and was found to be $29.6 \pm 16.7 \%$.

\section{Predictors of mortality}

Overall survival in the median observation period of 28 (95\% Cl: 12-41) days totaled 79 (33.6\%) patients, out of the 235 subjects. An overwhelming majority ( 95\%) of deaths were in-hospital events, in conjunction with the initial hospitalization.
Detailed results of our statistical analysis are shown in Table III. Univariate analysis of the Cox regression model showed that (I) prolonged VA-ECMO duration of more than 7 days, (II) body mass index $>30 \mathrm{~kg} / \mathrm{m}^{2}$, (III) ejection fraction < 30\%, (IV) male gender and (V) out-of-hospital CPR did not influence mortality outcomes in this patient population. Factors such as (I) age of the patient

Table II. Clinical and demographic data of patients $(n=235)$

\begin{tabular}{lc} 
Patient data & Value \\
\hline Age (mean \pm SD) [years] & $52.7 \pm 15.7$ \\
\hline Male gender, $n(\%)$ & $174(74)$ \\
\hline Cardiovascular risk factors, $n(\%):$ & $52(22.1)$ \\
\hline Diabetes mellitus & $50(21.2)$ \\
\hline Prior coronary revascularization & $48(20.4)$ \\
\hline Prior ACS & $59(25.1)$ \\
\hline Age $\geq 65$ years & $60(25.5)$ \\
\hline BMI > 30 kg/m & $32(13.6)$ \\
\hline Out-of-hospital CPR in conjunction with ECMO & $63(26.8)$ \\
\hline In-hospital CPR in conjunction with ECMO & $50.4 \pm 24.7$ \\
\hline GFR (mean \pm SD) [ml/min/1.73 m²] & $29.6 \pm 16.7$ \\
\hline Ejection fraction (mean \pm SD) $(\%)$ & $7.33 \pm 0.12$ \\
\hline pH value (mean \pm SD) & $7.83 \pm 5.98$ \\
\hline Lactate value (mean \pm SD) [mmol/l] & $79(33.6)$
\end{tabular}

Continuous parameters are expressed as average \pm standard deviation ACS - acute coronary syndrome, BMI - body mass index, CPR - cardiopulmo nary resuscitation, GFR - glomerular filtration rate.

Table III. Uni- and multivariate Cox models

\begin{tabular}{|c|c|c|c|c|}
\hline \multirow[t]{2}{*}{ Variables } & \multirow{2}{*}{$\frac{\text { Univariate analysis }}{P \text {-value }}$} & \multicolumn{3}{|c|}{ Multivariate analysis (stepwise backward final model) } \\
\hline & & $P$-value & Hazard ratio & $95 \% \mathrm{Cl}$ \\
\hline $\mathrm{pH}<7.3$ & $<0.001$ & $<0.001$ & 3.56 & $2.37-5.35$ \\
\hline Age $\geq 65$ years & 0.001 & 0.001 & 1.96 & $1.30-2.95$ \\
\hline Post-hTX indication & 0.015 & 0.025 & 0.51 & $0.29-0.92$ \\
\hline CS ACS indication & 0.002 & 0.073 & 1.44 & $0.97-2.14$ \\
\hline $\mathrm{GFR}<60 \mathrm{ml} / \mathrm{min} / 1.73 \mathrm{~m}^{2}$ & 0.031 & - & & \\
\hline In-hospital CPR & 0.007 & - & & \\
\hline Central cannulation & 0.014 & - & & \\
\hline Lactate > $3.3 \mathrm{mmol} / \mathrm{l}$ & 0.016 & - & & \\
\hline ECMO-VAD conv. & 0.022 & - & & \\
\hline $\mathrm{DM}$ & 0.041 & - & & \\
\hline ECMO time $>7$ days & 0.134 & - & & \\
\hline Out-of-hospital CPR & 0.189 & - & & \\
\hline $\mathrm{BMI}>30 \mathrm{~kg} / \mathrm{m}^{2}$ & 0.335 & - & & \\
\hline$E F<30 \%$ & 0.367 & - & & \\
\hline Male gender & 0.951 & - & & \\
\hline
\end{tabular}


$\geq 65$ years $(p<0.001)$, (II) GFR $<60 \mathrm{ml} / \mathrm{min} / 1.73 \mathrm{~m}^{2}$ ( $p=0.024)$, (III) in-hospital CPR $(p=0.003),(I V) \vee A-E C M O$ initiation due to CS ACS ( $p=0.003)$, (V) VA-ECMO initiation due to primary graft failure after hTX $(p=0.016)$, $(\mathrm{VI})$ central VA-ECMO cannulation $(p=0.029)$, (VII) VAECMO conversion to longer-term VAD systems $(p=0.034)$, (VIII) $\mathrm{pH}<7.3$ ( $p<0.001)$, (IX) lactate levels $>3.3 \mathrm{mmol} / \mathrm{l}$ and $(\mathrm{X})$ history of treated diabetes all exhibited probabilities of $p<0.1$ in the univariate model, of which three were found to be independent predictors of mortality after the backwards stepwise multivariate analysis. One factor displayed an opposite correlation, alas proving an independent factor for survival. Mortality predictors were (I) $\mathrm{pH}$ levels of less than $7.3(p<0.001, \mathrm{HR}=3.56$, 95\% Cl: 2.37-5.35), (II) age of the subject during ECMO care $\geq 65$ years $(p=0.001, \mathrm{HR}=1.96,95 \% \mathrm{Cl}: 1.3-2.95)$ and (III) VA-ECMO initiation due to CS ACS ( $p=0.073$, $H R=1.44,95 \% \mathrm{Cl}: 0.97-2.14)$. An independent factor for

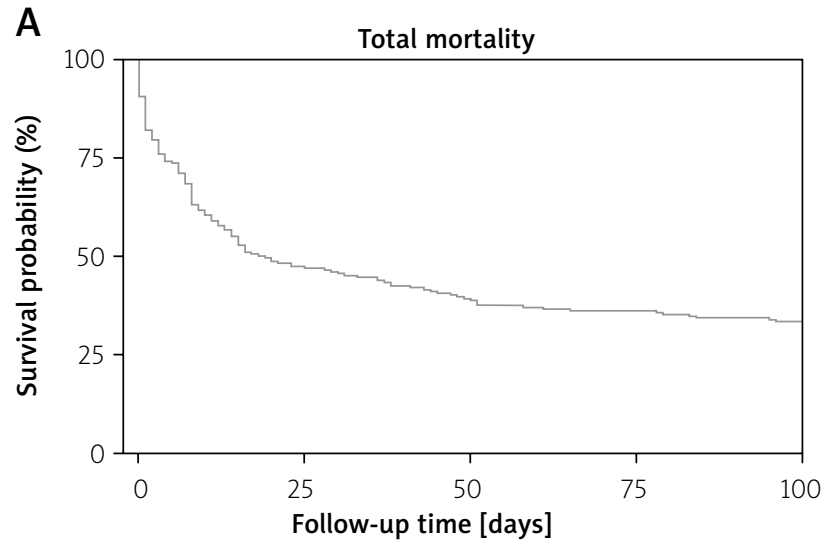

B
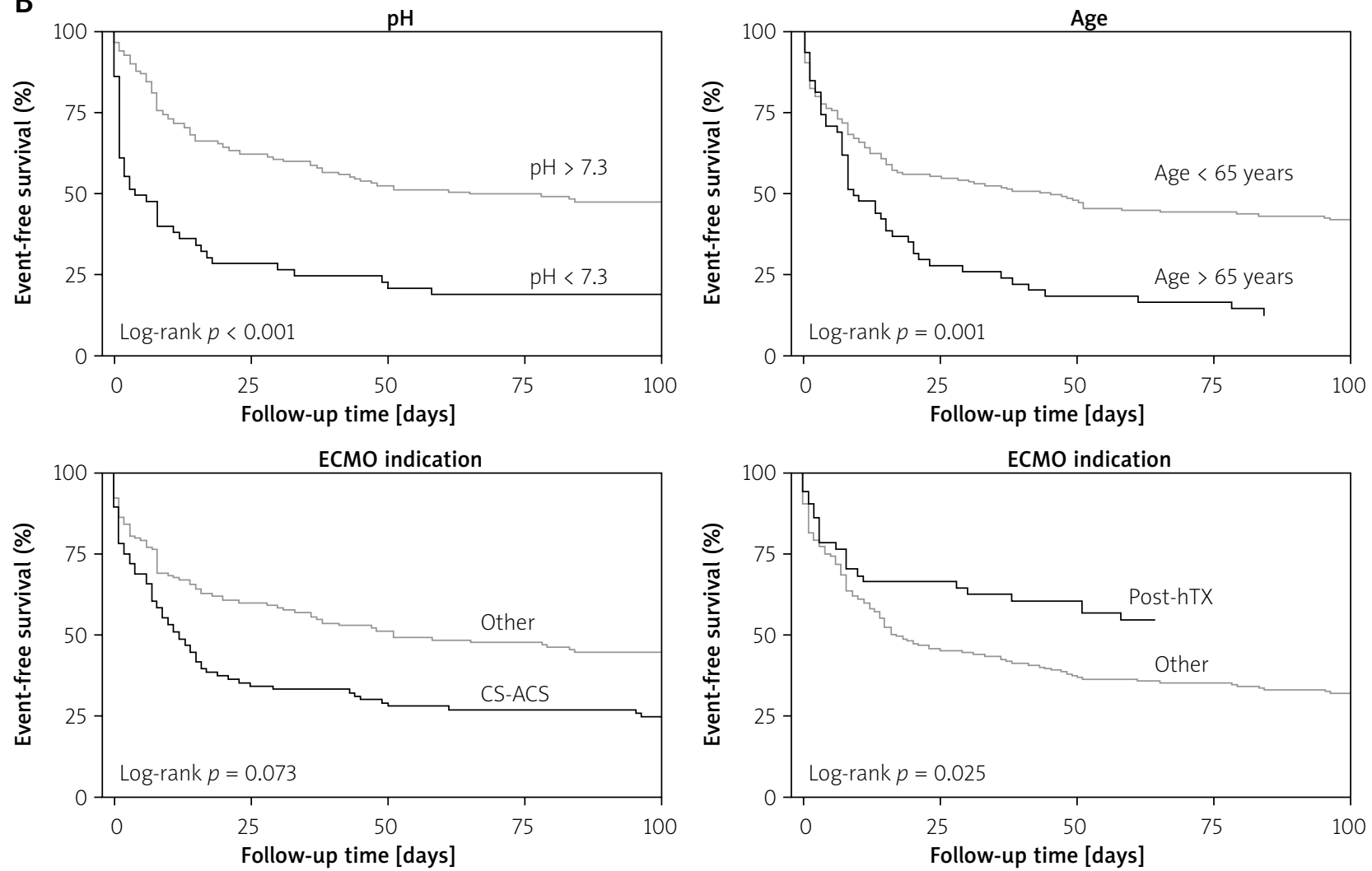

Figure 2. Kaplan-Meier curves displaying outcomes of venous-arterial extracorporeal membrane oxygenation (VA-ECMO) treatments. Total mortality of the study cohort is displayed in panel (A), showing a median follow-up of 28 days $(95 \% \mathrm{Cl}: 12-41)$. Survival plots of the patients were also examined with regards to parameters influencing mortality (B). Log-rank testing showed that $\mathrm{pH}$, age and post-heart transplantation (post-hTX) indication cause a significant difference in mortality, with $p$-values of $p<0.001, p=0.001$ and $p=0.025$, respectively. The effect of acute coronary syndromes related cardiogenic shock (CS-ACS) indication on mortality did not prove to be statistically significant 
survival proved to be VA-ECMO initiation due to primary graft failure after $\mathrm{hTX}(p=0.025, \mathrm{HR}=0.51,95 \% \mathrm{Cl}$ : 0.29-0.92). Kaplan-Meier survival graphs were plotted to display total mortality (Figure $2 \mathrm{~A}$ ) as well as survival with and without the three significant impactors of mortality and one impactor of survival (Figure 2 B).

\section{Discussion}

Our current study presents data from one of the largest single center databases to date. It shows that when VA-ECMO treatment is indicated, survival is negatively affected only by acidic $\mathrm{pH}$ values, advanced age and ACS as an indication for MCS, while hTX as an indication proved beneficial for survival.

\section{Unique representation of MCS patients}

VA-ECMO treatment is widely acknowledged as a systemic circulation augmentation technique, providing effective cardiac output for the short-term need. Implementation and use have extensively been discussed before $[1,3,14]$. Also, VA-ECMO treatment results in various underlying diseases have also been described in prior publications focusing on indications such as post-cardiotomy shock $[15,16]$, CS ACS $[17,18]$, and primary graft failure after hTX $[19,20]$, albeit all in relatively small registries. The results were most favorable in hTX cases, while they were found to be poor in CS ACS. Outcomes of post-cardiotomy patients requiring VA-ECMO treatment varied within these boundaries. Treatment results and outcomes for the respective groups were comparable in the SAVE study [8].

Our results are unique in the field as the data are derived from an extensive, real-world, all-comers cohort of a single, national level VA-ECMO center. We opted to include every patient undergoing VA-ECMO treatment and focus on the treatment outcomes themselves as a method to investigate parameters that favorably or adversely influence mortality, instead of selecting a subpopulation of our patient cohort to analyze. Thus, we achieved one of the largest single center registries to date. As shown in numerous other prior studies [3, 8, 15-20], patients in need of VA-ECMO care regardless of any other clinical parameter present as extremely high-risk individuals. Our survival rate of $33.5 \%$ correlates with previous data [21], and although mortality remains consistently high across the spectrum, VA-ECMO treatment definitely saves lives that would otherwise be lost.

\section{VA-ECMO indications: hTX and CS ACS, $\mathrm{pH}$ levels and age}

Our results showed that mortality of patients requiring VA-ECMO support is only partially influenced by the type of initial cardiovascular disease associated with the need for MCS. As with already available results, the only underlying medical MCS indication negatively affecting mortality proved to be ACS and CS. Similarly, MCS indication following hTX independently assured a more positive outcome for subjects. Our data are partially in accordance with published findings, in which a definite trend was also detectable [8, 15-20]. During hTX surgery, post-operative patients are closely monitored, and if the need for higher level circulatory support arises, this may be initiated early, without significant prolongation of low-cardiac output times. Hence, disruption of the metabolic and respiratory equilibrium can be kept to a minimum.

Reliable data are also available indicating that in suitable patients, VA-ECMO treatment in ACS associated CS improves survival, as compared with non-MCS therapy [22]. With regards to our VA-ECMO database, we assessed ACS in conjunction with CS of INTERMACS level 1 patients to determine the impact on mortality. CS ACS statistically ( $p=0.073, \mathrm{HR}=1.44,95 \% \mathrm{Cl}$ : $0.97-$ $2.14)$ provided an increased hazard ratio, with a $p$-value of near statistical significance, displaying a measurable negative impact on survival. As CS ACS patients mostly require lengthy transport to destination therapy institutions, duration of shock tends to be prolonged, which allows the process to progress beyond reversibility, thereby decreasing the chance of survival and leading to worse outcomes. Assessing the aforementioned data led us to infer that, primarily, not the initial cardiovascular disease resulting in CS, but rather the extent and reversibility of metabolic derailment, depth of shock, the availability of physiological reserves and general condition of patients influence outcomes. Accordingly, reduced $\mathrm{pH}$ levels at MCS initiation proved to be a clear predictor of mortality in our cohort, as seen before in the SAVE database $[8,23]$. The development of lactate acidosis and consequent drop in $\mathrm{pH}$ develops quickly in CS and strongly correlates with the severity of shock and thus mortality. Similarly, advanced age is known to negatively impact almost every medical outcome, including invasive procedures, especially in cardiovascular diseases. CS mortality increases with advancing age [24, 25], irrespective of treatment or counterpulsation use. Our data confirm these results as we also observed markedly worse outcomes in patients $\geq 65$ years of age compared with younger individuals (Table III). In the current population, mortality of patients above 65 years of age undergoing VA-ECMO treatment was close to $90 \%$, which might be expected as older patients exhibit decreased physiological reserves, a wider extent of co-morbidities, generally increased frailty and thus higher susceptibility to low cardiac output conditions, resulting in early irreversible multi-organ failure and death. Surviving elderly individuals shared a similar clinical course: a sudden circulatory collapse and/or CPR need immediately treated with advanced life support (ALS) during an invasive procedure (mainly percutaneous coronary intervention), after which the peripheral 
VA-ECMO system was successfully brought online within 10-15 min, keeping CS duration to a minimum and so increasing survival.

\section{Parameters without a significant effect on mortality}

Based on the analysis of our database, our observation is that the effect of parameters generally accepted to be relevant with regards to cardiovascular mortality and decreased survival, such as diabetes, reduced ejection fraction, prior ACS or coronary revascularization, are not statistically significant independent predictors of mortality in our real-world population of patients in CS. Other research groups investigating comparable populations published similar results [26-28]. Also, we did not observe differences in mortality outcomes in conjunction with the need for CPR. We included both in-hospital cardiac arrest (IHCA) and OHCA cases in the database. As reliable data for the exact return of spontaneous circulation (ROSC) and/or habitual CPR were often lacking especially in OHCA cases - such stratification was not undertaken. Yet, as all cases following CPR presented either as non-ROSC/habitual CPR need or ROSC but at an INTERMACS level 1, we can safely state that both patient subgroups (OHCA and IHCA) were at all times in a critical condition. Surprisingly however, serum lactate levels did not prove to be a prognostic factor. Causes in the background of these findings may prove difficult to explain, although it is reasonable to assume that our data shows that after circulatory collapse, especially coupled with CPR need, lactate levels increase exponentially over time, but also drop quickly after ROSC, displaying rapid shifts in level characteristics. Yet, $\mathrm{pH}$ levels only start decreasing after an initial phase of compensated acidosis. Thus, a drop in $\mathrm{pH}$ is much more strongly associated with the extent of metabolic derailment and expense of reserves, clearly indicating potential death at an acidic level $(\mathrm{pH}<7.3)$. This is exceptionally true for CPR cases, in which metabolic exhaustion happens at an increased pace and death is imminent without the fastest possible MCS treatment.

Furthermore, it is reasonable to assume that the traditional risk factors of cardiovascular mortality do not influence outcomes of these patients since they require more time, usually several months or years, to establish a measurable impact. In contrast, most of the deaths in this population occurred during initial hospitalization in the first couple of days or weeks following VA-ECMO cannulation (Figure 2).

In a similar fashion, prolonged VA-ECMO duration, system re-cannulation and upgrade to VAD systems also failed to influence mortality alone, as the metabolic (and thus mortality) impact necessitating the above-mentioned extensions of MCS therapy manifested prior to the interventional or surgical upgrade need.

\section{Conclusions}

Our database shows that the VA-ECMO perfusion system can successfully be applied to a large cohort of critically ill CS subjects irrespective of the initial disease. In our registry, mortality outcomes were negatively influenced by advanced age, low $\mathrm{pH}(<7.3)$ and the need for MCS due to ACS. Indication for MCS in an hTX setting proved beneficial for survival. Accounting for all the above, the depth and severity and hence the reversibility of CS at VA-ECMO initiation depended upon advanced age and an acidic environment $(\mathrm{pH}<7.3)$ and might also be influenced by extended circulatory insufficiency in conjunction with ACS transport times. Age and decreased $\mathrm{pH}$ negatively impact survival of patients according to our data. With this relevant clinical information at hand we have a better understanding of outcome-influencing factors at the time of VA-ECMO initiation. Nonetheless, as most reported data are from registries and retrospective analyses, further prospective and ideally randomized studies are required to verify findings thus far and assess outcomes in detail.

\section{Conflict of interest}

The authors declare no conflict of interest.

\section{References}

1. Lafç G, Budak AB, Yener AÜ, Cicek OF. Use of extracorporeal membrane oxygenation in adults. Heart Lung Circ 2014; 23: 10-23.

2. Cavarocchi NC, Wallace S, Hong EY, et al. A cost-reducing extracorporeal membrane oxygenation (ECMO) program model: a single institution experience. Perfusion 2015; 30: 148-53.

3. Mosier JM, Kelsey M, Raz Y, et al. Extracorporeal membrane oxygenation (ECMO) for critically ill adults in the emergency department: history, current applications, and future directions. Crit Care 2015; 19: 431.

4. Gu K, Zhang Y, Gao B, et al. Hemodynamic differences between central ECMO and peripheral ECMO: a primary CFD study. Med Sci Monit 2016; 22: 717-26.

5. Kanji HD, Schulze CJ, Oreopoulos A, et al. Peripheral versus central cannulation for extracorporeal membrane oxygenation: a comparison of limb ischemia and transfusion requirements. Thorac Cardiovasc Surg 2010; 58: 459-62.

6. Toda K, Fujita T, Seguchi O, et al. Role of percutaneous veno-arterial extracorporeal membrane oxygenation as bridge to left ventricular assist device. J Artif Organs 2018; 21: 39-45.

7. Marasco SF, Lo C, Murphy D, et al. Extracorporeal life support bridge to ventricular assist device: the double bridge strategy. J Artif Organs 2016; 40: 100-6.

8. Schmidt M, Burrell A, Roberts L, et al. Predicting survival after ECMO for refractory cardiogenic shock: the survival after veno-arterial-ECMO (SAVE)-score. Eur Heart J 2015; 36: 2246-56.

9. Abrams D, Combes A, Brodie D. Extracorporeal membrane oxygenation in cardiopulmonary disease in adults. J Am Coll Cardiol 2014; 63: 2769-78

10. Stevenson LW, Pagani FD, Young JB, et al. INTERMACS profiles of advanced heart failure: the current picture. J Heart Lung Transplant 2009; 28: 535-41. 
11. Lamb KM, DiMuzio PJ, Johnson A, et al. Arterial protocol including prophylactic distal perfusion catheter decreases limb ischemia complications in patients undergoing extracorporeal membrane oxygenation. J Vasc Surg 2017; 65: 1074-79.

12. Juo YY, Skancke M, Sanaiha Y, et al. Efficacy of distal perfusion cannulae in preventing limb ischemia during extracorporeal membrane oxygenation: a systematic review and meta-analysis. Artif Organs 2017; 41: E263-73.

13. Lamb KM, Hirose $H$. Vascular complications in extracoporeal membrane oxygenation. Crit Care Clin 2017; 33: 813-24.

14. Allen S, Holena D, McCunn M, et al. A review of the fundamental principles and evidence base in the use of extracorporeal membrane oxygenation (ECMO) in critically ill adult patients. J Intensive Care Med 2011; 26: 13-26.

15. Miana LA, Canêo LF, Tanamati C, et al. Post-cardiotomy ECMO in pediatric and congenital heart surgery: impact of team training and equipment in the results. Rev Bras Cir Cardiovasc 2015; 30: 409-16.

16. Khorsandi M, Shaikhrezai K, Prasad S, et al. Advanced mechanical circulatory support for post-cardiotomy cardiogenic shock: a 20-year outcome analysis in a non-transplant unit. J Cardiothorac Surg 2016; 11: 29.

17. Negi SI, Sokolovic M, Koifman E, et al. Contemporary use of veno-arterial extracorporeal membrane oxygenation for refractory cardiogenic shock in acute coronary syndrome. J Invasive Cardiol 2016; 28: 52-7.

18. Pavasini R, Cirillo C, Campo G, et al. Extracorporeal circulatory support in acute coronary syndromes: a systematic review and meta-analysis. Crit Care Med 2017; 45: e1173-83.

19. Lima EB, Cunha CR, Barzilai VS, et al. Experience of ECMO in primary graft dysfunction afterorthotopic heart transplantation. Arq Bras Cardiol 2015; 105: 285-91.

20. Marasco SF, Vale M, Pellegrino V, et al. Extracorporeal membrane oxygenation in primary graft failure after heart transplantation. Ann Thorac Surg 2010; 90: 1541-6.

21. Rao P, Khalpey Z, Smith R, et al. Venoarterial extracorporeal membrane oxygenation for cardiogenic shock and cardiac arrest. Circ Heart Fail 2018; 11: e004905.

22. Harjola VP, Lassus J, Sionis A, et al. Clinical picture and risk prediction of short-term mortality in cardiogenic shock. Eur J Heart Fail 2015; 17: 501-9.

23. Fuernau G, Poenisch C, Eitel I, et al. Prognostic impact of established and novel renal function biomarkers in myocardial infarction with cardiogenic shock: a biomarker substudy of the IABP-SHOCK II-trial. Int J Cardiol 2015; 191: 159-66.

24. Schwarz B, Abdel-Wahab M, Robinson DR, Richardt G. Predictors of mortality in patients with cardiogenic shock treated with primary percutaneous coronary intervention and intra-aortic balloon counterpulsation. Med Klin Intensivmed Notfmed 2016; 111: 715-22.

25. Pineton de Chambrun $M$, Bréchot N, Combes A. Venoarterial extracorporeal membrane oxygenation in cardiogenic shock: indications, mode of operation, and current evidence. Curr Opin Crit Care 2019; 25: 397-402.

26. Fux T, Holm M, Corbascio M, et al. VA-ECMO support in nonsurgical patients with refractory cardiogenic shock: pre-implant outcome predictors. Artif Organs 2019; 43: 132-41.

27. D'Arrigo S, Cacciola S, Dennis M, et al. Predictors of favourable outcome after in-hospital cardiac arrest treated with extracorporeal cardiopulmonary resuscitation: a systematic review and meta-analysis. Resuscitation 2017; 121: 62-70.
28. Debaty G, Babaz V, Durand M, et al. Prognostic factors for extracorporeal cardiopulmonary resuscitation recipients following out-of-hospital refractory cardiac arrest. A systematic review and meta-analysis. Resuscitation 2017; 112: 1-10. 